Meta

Journal des tradlucteurs

Translators' Journal

\title{
Pour un modèle rétroactif d'enseignement de la traduction générale anglais-français
}

\section{Jean-Marc Gouanvic}

Volume 33, numéro 2, juin 1988

L'enseignement de la traduction au Canada - Teaching Translation in Canada

URI : https://id.erudit.org/iderudit/002952ar

DOI : https://doi.org/10.7202/002952ar

Aller au sommaire du numéro

Éditeur(s)

Les Presses de l'Université de Montréal

ISSN

0026-0452 (imprimé)

1492-1421 (numérique)

Découvrir la revue

Citer cet article

Gouanvic, J.-M. (1988). Pour un modèle rétroactif d'enseignement de la traduction générale anglais-français. Meta, 33(2), 156-163.

https://doi.org/10.7202/002952ar d'utilisation que vous pouvez consulter en ligne.

https://apropos.erudit.org/fr/usagers/politique-dutilisation/ 


\title{
POUR UN MODÈLE RÉTROACTIF D'ENSEIGNEMENT DE LA TRADUCTION GÉNÉRALE ANGLAIS-FRANÇAIS
}

\author{
JEAN-MARC GOUANVIC \\ Université du Québec à Trois-Rivières, Québec
}

\section{INTRODUCTION}

Il existe aujourd'hui quelques bons ouvrages sur la pédagogie de la traduction générale (anglais-français) et le professeur dispose d'outils bien adaptés à sa tâche. Le livre de Jean-Paul Bénard et Paul Horguelin ${ }^{1}$, ainsi que celui de Jean Delisle ${ }^{2}$, pour ne citer que ces deux-là, constituent de par leurs visées propres des ouvrages intéressants pour notre propos. Je n'insisterai pas sur les éléments que recoupent ces livres même si, chacun à leur manière, ils ont contribué à l'élaboration de la pratique pédagogique dont il va être ici question.

Ce qui caractérise le cours "Traduction générale - anglais-français ", c'est d'abord qu'il est le plus souvent le premier cours de traduction offert dans nos programmes. Constatation lourde de conséquences : c'est dans ce cours que l'apprentitraducteur va apprendre ce que traduire veut dire, qu'il va être mis en contact avec l'activité de traduction, la discipline qu'elle exige, et sa déontologie même. Le futur traducteur est dans ce cours censé acquérir une partie du jugement nécessaire pour évaluer la marge de manœuvre dont il dispose entre le message de la langue de départ (LD) et celui de la langue d'arrivée (LA). C'est dans ce cours qu'il acquerra certains réflexes qui pourront faire de lui un vrai professionnel.

Nous ne nous appesantirons pas sur les lacunes de nos étudiants : lacunes de compréhension de la LD, lacunes dans la simple connaissance du code linguistique de la LA ; il appartient à chaque programme d'établir la limite de compétence linguistique de chaque étudiant à son admission. Il n'est d'ailleurs pas sûr que la situation soit aussi catastrophique qu'on le dit parfois. Selon certains employeurs, une partie appréciable des étudiants diplômés font de très honnêtes traducteurs, même s'ils ne deviendront de véritables professionnels qu'après des mois sinon des années de pratique intensive, notamment sous la direction de collègues chevronnés ou de réviseurs.

Un cours de traduction générale vise d'abord à inculquer au débutant les mécanismes essentiels de l'opération de traduction, ces mécanismes mêmes qu'un traducteur autodidacte mettra sans doute plus de temps à acquérir, parce qu'il procède par tâtonnements. Nous présenterons les éléments clés de la pratique pédagogique telle que nous la concevons de façon tout à fait concrète, en fonction des objectifs visés. C'est cette pratique pédagogique qu'il a semblé pertinent de nommer "rétroactive "3. Qu'il soit immédiatement entendu qu'il ne s'agit là que d'une façon parmi d'autres de former de jeunes traducteurs.

\section{PRÉLIMINAIRES}

Rappelons brièvement les principes qui président au bon déroulement de ce cours avant d'en arriver aux points centraux sur lesquels nous concentrerons notre attention. 
1. Le cours de traduction générale se trouve, à l'Université du Québec à TroisRivières, à la confluence de quatre autres cours dispensés la même année :

- Grammaire différentielle de l'anglais et du français (ou Stylistique comparée) ;

- Canadianismes et anglicismes (ou Québécismes et anglicismes);

- Rédaction et style (ou Stylistique et grammaire du français écrit) ;

- Lexique et traduction (cours conçu dans l'optique de l'analyse du discours en français avec des lectures dirigées et des études de textes).

(Ce cours est nouveau dans sa conception au sein du programme de traduction.)

L'enseignement de la traduction générale est très largement tributaire de ces quatre cours, sur lesquels il s'appuie ; le professeur établit des relations avec ces derniers chaque fois que cela est possible et en rappelant au besoin le métadiscours propre à chacun d'entre eux.

2. Comme il s'agit d'un premier cours de traduction, le professeur présente un panorama de la profession de traducteur au Québec et au Canada, en fournissant des bibliographies de base sur la profession ainsi que sur les professions connexes (terminologue, réviseur, etc.).

3. Les étudiants n'ayant en général aucune idée, d'une part, des difficultés qui les attendent et, d'autre part, de ce que l'on peut considérer comme une traduction professionnellement acceptable, des critères d'une bonne traduction leur sont proposés. Ces critères, ou paramètres, sont sous leur forme simplifiée classés en cinq catégories : exactitude dans le transfert sémantique, la correction linguistique en LA, la transparence en LA, le respect en LA du niveau de langue et de la tonalité de la LD, l'adaptation au destinataire 4

Ainsi, les étudiants disposent d'emblée de paramètres qui ont comme avantage de maintenir une interaction raisonnée entre le texte en LD et le texte en LA, et entre ce que le professeur attend de l'étudiant et ce que l'étudiant attend du professeur. Toute pédagogie exige cette interaction que permet le cadre théorico-pratique des paramètres. Plus loin, nous verrons comment ce cadre sert également à l'évaluation et à la notation.

4. Choix des textes. Le cours ne doit pas donner lieu à des recherches à caractère terminologique ; il doit être principalement axé sur les mécanismes de transfert sémantique et de remodelage syntaxique. Les textes seront donc choisis en fonction de ces visées et dans les domaines de la culture générale. Cela signifie en pratique que tout sujet est considéré comme adapté au cours. Toutefois, nous considérons comme profitable la lecture d'un article d'encyclopédie générale (Universalis, par exemple) pour se remettre en mémoire des détails de connaissance générale et pour capter l'esprit d'un texte sur un sujet connexe écrit en LA. Il est loin d'être inutile de consulter un article d'encyclopédie sur Léonard de Vinci si l'on a à traduire un texte où il est question du génie universel du Florentin ou, comme dans le texte que nous avons choisi, sur la révolution industrielle.

5. Quels que soient les textes choisis, le professeur oriente le travail des étudiants en replaçant le texte dans son contexte de publication (ce qui correspond au paramètre 4), en indiquant les éléments du texte qui renseignent sur le public visé (paramètre 5). II oriente aussi les choix traductionnels des étudiants en indiquant pourquoi il a choisi ce texte plutôt qu'un autre : travail de saisie sêmantique lié aux formulations en LD (paramètre 1) ; remodelage syntaxique approfondi en LA (paramètre 2 ) ; risques d'anglicismes en LA (paramètre 3); travail stylistique particulier pour obtenir la meilleure transparence en LA (paramètre 3) ; travail sur les niveaux de langue et les tonalités (paramètre 4) ; travail d'adaptation au destinataire (paramètre 5). Il est évident qu'un texte peut comporter de nombreux genres de traquenards. C'est en fin de session que les étudiants pourront être confrontés à plusieurs d'entre eux d'une façon que l'on pourrait 
qualifier de " sauvage ». En attendant, il faut calibrer les difficultés en s'efforçant de traiter la matière de façon progressive et veiller à ce que l'essentiel soit compris.

\section{QU'EST-CE QUE TRADUIRE?}

Dès le début, il est important que les étudiants aient une idée de ce qu'est la traduction. Le meilleur moyen, nous semble-t-il, est de leur proposer quelques phrases simples à traduire. Ainsi pourront-ils s'interroger sur une opération qu'ils viennent de pratiquer et sur laquelle il leur suffira de revenir intellectuellement en en analysant les processus. Il est essentiel que les énoncés proposés forment des contextes suffisants (sinon, le professeur doit fournir des données contextuelles complètes) et ne présentent pas de difficultés insurmontables.

Le professeur inscrit l'énoncé suivant :

On Tuesday, October 26, 1948, the people in Donora, a small industrial town in Pennsylvania, woke up to discover that they were trapped in a dense pall of smog drenched with odoriferous chemicals.

Les étudiants ont quelques minutes pour proposer une traduction spontanée, sans l'aide d'aucun document. Tous les mots de cet énoncé sont sans doute connus, sauf un : pall. Les étudiants sont priés de tenter une traduction. Il est remarquable qu'un certain nombre d'entre eux parviennent à des solutions acceptables, même s'ils sont de véritables débutants. Bien sûr, certains anglicismes ont la vie dure (présentation de la date, apposition entre virgules) ; mais quand on peut d'emblée faire appel à leur jugement linguistique, ils savent assez vite trouver la logique sémantique de cet énoncé : people/population, drenched/imprégné, odoriferous/nauséabond. Quant à dense pall, il est demandé aux étudiants de trouver le mot le plus « naturel » en français pour désigner cette concentration de smog (il n'est pas question à ce stade de parler de cooccurrence); nappe, voile sont mentionnés. Une, deux ou même trois traductions sont retranscrites au tableau, puis commentées. Il reste un point à régler dans la traduction de cette phrase : l'ordre des actions dans woke up to discover. Une étudiante, à juste titre bien entendu, se déclare gênée par se réveilla pour découvrir. De fait, le français opère ici une interversion (une modulation), mais encore une fois il est prématuré de faire référence en début de session à l'appareil conceptuel de la Stylistique comparée : la population de Donora découvrit en se réveillant/à son réveil.

Au bout d'un quart d'heure d'échanges, les étudiants sont parvenus à une traduction tout à fait satisfaisante. Le professeur classe alors les problèmes rencontrés par catégories : saisie du sens de l'énoncé, problèmes syntaxiques liés ou non à l'anglicisation, problèmes stylistiques proprement dits, niveaux de langue, etc.

Ces catégories peuvent être réunies en deux groupes principaux : les questions qui relèvent du transfert sémantique et celles qui sont liées à la "forme" du message en français. Le professeur peut alors avancer une définition de ce qu'est traduire. Par exemple : " La traduction consiste à produire dans la langue d'arrivée l'équivalent naturel le plus proche du message de la langue de départ, d'abord quant à la signification, puis quant au style. " On a reconnu la célèbre définition proposée par Eugène Nida.

Le professeur peut trouver profitable de multiplier les exercices de ce genre. Il semble en effet souhaitable de mettre les étudiants en présence de contextes divers. J'ai, par exemple, soumis aux étudiants cette phrase extraite d'un article écrit par un psychiatre : The importance of treating each person individually is obvious. (Le professeur doit indiquer qu'il s'agit d'un traitement, par la technique des entrevues notamment.)

Les traductions proposées par les étudiants sont les suivantes :

a) Il est important de traiter chaque personne de façon individuelle. 
b) Il est évidemment important de traiter chaque personne individuellement.

c) Il est évident que c'est important de traiter chaque personne individuellement.

d) L'importance de traiter chaque personne individuellement est évidente.

e) Il est évident que traiter chaque personne individuellement est important.

f) Le traitement individuel de chaque personne est évidemment important.

g) Traiter chaque personne individuellement est évidemment important.

h) Il est évident qu'il faut traiter chaque personne individuellement.

La traduction d'une phrase aussi simple permet de dégager les aspects suivants :

- L'angle d'attaque en français est de préférence fourni par une formule impersonnelle du genre «il est évident » (fait stylistique).

- La répétition des adverbes de manière en -ment n'est pas souhaitable (fait styHistique).

- Dans la formulation C, «c'est important de » est d'un niveau de langue qui ne convient pas à l'écrit.

- La formulation A comporte une lacune sémantique : omission de la qualification "obvious".

- La formulation $\mathrm{H}$ est sans doute attrayante, mais elle contient un léger glissement de sens contre lequel il convient de mettre les étudiants en garde : "The importance of " ne peut être exactement traduit par « il faut ». "Importance » ne dénote pas la forte prescription que comporte nécessairement " il faut ".

- Enfin, il est à noter que la proposition $\mathrm{D}$, parfaitement symétrique de la formulation anglaise, est également acceptable, quoique peu idiomatique.

La formulation A est stylistiquement heureuse et il suffit d'ajouter " évidemment " après « il est " pour corriger la lacune sémantique. De légères corrections (par exemple, remplacer "évidemment " de la phrase G par un adverbe non terminé en -ment comme "de toute évidence ") suffisent pour rendre plusieurs de ces formulations stylistiquement et sémantiquement correctes. Cet exercice a un avantage supplémentaire : il montre qu'il n'existe pas qu'une solution aux problèmes de traduction, même si en général certaines formulations emportent l'adhésion des traducteurs et des apprentistraducteurs mêmes. Sur une phrase comme celle-là, il est particulièrement facile de faire ressortir le travail d'analyse sémantique en contexte : le flou de "treating " pris hors contexte (" traiter " ou " considérer "?) en est un exemple patent, même s'il fonctionne $a$ contrario. Ce travail d'analyse est bien entendu à rapprocher de la recherche des unités de traduction que les étudiants étudieront dans les semaines qui suivent au cours de Grammaire différentielle.

\section{LES TRAVAUX PRATIQUES}

Ce cours d'introduction à l'exercice de la traduction est un apprentissage pratique. Cela signifie qu'il apparaît prioritaire que les étudiants fassent de nombreux travaux pratiques chez eux et que leur progression soit évaluée par des tests en temps limité. À 15 séances de 3 heures correspondent 7 TP et 3 tests en classe. Les TP aussi bien que les tests font entre 200 et 250 mots.

Au deuxième cours est longuement présentée la manière d'aborder un texte à traduire ${ }^{5}$. À la fin de ce cours, les étudiants reçoivent leur premier TP à faire à domicile pour la semaine suivante. Le professeur présente le texte pour orienter la traduction des étudiants. En outre, ces derniers reçoivent comme directive de dactylographier leur traduction à double ou triple interligne sans rature ni retouche à la main.

La semaine suivante est effectuée la correction collective du TP. Un étudiant présente son cheminement sur la première phrase : il analyse les difficultés qu'il a rencontrées, de quelque ordre qu'elles soient (saisie du sens, faux amis, syntaxe alambiquée, passages ambigus, etc.) et indique comment il les a résolues. Le professeur intervient dès 
cet instant pour souligner la pertinence ou la non-pertinence des remarques de l'étudiant (par exemple, difficultés intuitivement aperçues, mais erreur sur leur nature, et donc solution proposée généralement non satisfaisante). À ce stade, le professeur ne corrige pas les erreurs ni, a fortiori, ne propose de traduction. Il suscite les réactions des autres étudiants aux commentaires du premier étudiant : c'est alors que sont rectifiées les lacunes de la première traduction; puis d'autres questions sont posées par ceux et celles qui en manifestent le désir.

Les étudiants apprennent très vite qu'il n'y a pas qu'une traduction possible pour un même message : le professeur fait ressortir les bonnes traductions ; il peut les inscrire au tableau si cela peut s'effectuer rapidement. C'est l'occasion de faire apparaitre très clairement l'acquis des quatre cours de soutien en indiquant les procédés de traduction (modulation, étoffement, etc.), les formulations anglicisées, etc.

Pendant cette phase de commentaire critique, de correction et de choix des bonnes traductions, la rétroaction doit être réelle : les étudiants sont mis devant la nécessité de rectifier leur propre traduction lorsque cela est indispensable. Sur leur copie ils inscrivent leur correction dans l'interligne supérieur.

Lorsque le texte a été intégralement commenté et corrigé, les étudiants remettent leurs travaux au professeur qui sera ainsi en mesure de noter la version dactylographiée, qui correspond au travail fait à domicile, et de contrôler les corrections apportées par l'étudiant dans l'interligne en s'assurant que l'exercice a été bien compris.

Tout exercice d'autocorrection est certes difficile, surtout pour des débutants, et il ne serait guère souhaitable si le professeur n'avait pas les moyens de le contrôler et de rectifier les erreurs inévitables. L'autocorrection ne permet pas au professeur de faire l'économie d'une correction serrée des copies des étudiants. Bien au contraire, il s'agit pour lui de corriger deux travaux en un : le TP dactylographié et la correction qui en a été faite en classe par l'étudiant. Lorsque cette dernière a été bien réalisée, elle donne droit à des points en prime.

Mais il y a un autre avantage à cette méthode. Le professeur corrige le TP des étudiants en renvoyant dans la marge aux paramètres dont il a déjà été question $(\mathbf{P} 1, \mathbf{P} 2$, $\mathrm{P} 3$, etc.) et qui correspondent à une typologie simple des fautes. Lorsque les étudiants reçoivent leurs copies, le professeur leur demande de regrouper les paramètres par catégories. Ainsi, chaque étudiant peut d'un simple coup d'œil aux catégories percevoir ses points faibles et ses points forts. En dernier lieu, l'étudiant contrôle la notation en attribuant à chaque erreur un nombre de points qui sont soustraits de 100 pour donner la note du TP. Ce contrôle prend environ cinq minutes par cours.

\section{Exemple de TP}

(Début de session)

The Introduction of Industrial Research

Until about the middle of the 19th century it was the inventor rather than the scientist who was responsible for technical advance. This was partly because a certain maturity of growth is required of the fundamental sciences like physics and chemistry before they are in a position to contribute systematically, on a broad front, to ind ustrial progress. A further reason was the lack of scientific training among those responsible for running the industries.

This was particularly the case in Britain. Most of the industrially important inventions were at first British since Britain was the pioneer in the industrial revolution (an additional reason may have been that the patent system in England, as reformed by the act of 1624, gave a clear incentive to inventors and removed the basis of princely favours rather than technical innovation). To reduce their dependence on British technical knowledge other European countries notably France, Germany and Switzerland, established polytechnics and other technical schools during the first half of the 19th century. By the 1850s it was apparent that the resul- 
ting systematic application of science to industry was successful in producing technical advances.

Si la traduction est un décodage, elle est aussi, et surtout, un encodage. La pierre d'achoppement dans tous les cas de traduction digne de ce nom est la mise en français, l'emploi des idiotismes appropriés pour atteindre à la meilleure transparence (pour reprendre le concept de Georges Mounin) du message en LA.

Il a été demandé aux étudiants de faire une lecture active du texte, c'est-à-dire le crayon à la main, pour que les problèmes apparaissent immédiatement lors de la relecture au moment de passer à la traduction. Cette première lecture doit permettre au traducteur de saisir la ou les tonalités, le ou les niveaux de langue en $\mathrm{LD}^{5}$.

Le texte choisi est extrait d'un article d'encyclopédie sur l'histoire de la recherche scientifique; il ne comporte aucune idiosyncrasie d'auteur; il est d'un niveau de langue moyen et de nature informative.

Question 1 : Un étudiant (étudiant $\mathrm{A}$ ) répond aux questions suivantes :

"Dans la première phrase quelles sont les difficultés que vous avez rencontrées? "

Autrement dit, "Quels termes ou tournures (syntagmes) avez-vous soulignés ?"

Réponse : "J'ai souligné technical advance. Le dictionnaire me donne le choix entre avancement, progrès et développement. J'opte pour développement technique."

Le professeur suscite les commentaires des autres étudiants : un étudiant (B) dit avoir rencontré la forme avancement des sciences, mais ne parvient pas à retrouver de contexte. Le professeur précise que, de fait, l'expression est attestée au moins dans la désignation officielle de l'ACFAS : Association canadienne française pour l'avancement des sciences. Le professeur note que rien ne s'opposerait à l'emploi d'avancement des techniques. Cependant, il convient ici d'opter pour une formulation plus courante : développement technique ou progrès technique(s). Le professeur en profite pour noter l'occurrence commune d'avancement dans le contexte de travaux en voie de réalisation (cooccurrence des termes dans avancement des travaux).

Question 2 (posée à l'étudiant A) : La syntaxe de cette première phrase vous paraît-elle sans particularités? L'étudiant A ne voit pas. Un étudiant (C) remarque la mise en relief it was ... who .... Le professeur énonce la phrase sans la mise en relief (suppression de it was ... who ...). Il remarque qu'il s'agit d'une formulation conforme au gallicisme c'est ... qui ..., beaucoup moins usité en anglais sous cette forme.

Question 3 (posée à l'ensemble du groupe) : Y a-t-il d'autres remarques à faire sur cette phrase?

Réponse : Non. Le professeur se tourne alors vers l'étudiant A pour connaître sa traduction (à laquelle il aura intégré les remarques faites pendant l'échange) :

Jusque vers le milieu du $19^{\mathrm{e}}$ siècle, c'était l'inventeur plutôt que le scientifique qui était responsable du développement technique.

Jusqu'alors, le professeur n'a pas proposé de formulation ; il s'est contenté de "mettre de l'ordre" dans les suggestions des étudiants, rectifiant les erreurs, soulignant la justesse de certaines remarques. Certes, il peut arriver - et c'est assez souvent le cas - que la traduction collective des étudiants soit en tous points satisfaisante et qu'il n'y ait rien à ajouter. Dans la phrase que nous venons de traduire, quelques améliorations peuvent être apportées pour parfaire la transparence du message en LA.B

1. Il est approprié ici d'employer le présent historique pour traduire le prétérit anglais.

2. Même si l'emploi du concept de responsabilité dans ce contexte peut à la rigueur se défendre, il est préférable de traduire le véritable «vouloir-dire » de 
responsible for dans ce contexte : l'inventeur est l'artisan des progrès techniques.

3. Rather than se traduira en français de préférence par plus que (idiotisme).

4. Il existe un choix entre l'emploi du singulier et du pluriel dans le cas de progrès technique(s). D'où la traduction proposée par le professeur :

Jusque vers le milieu du $19 \mathrm{e}$ siècle, c'est l'inventeur plus que le savant qui est l'artisan des progrès techniques.

\section{Autre proposition :}

Jusque vers le milieu du 19 e siècle, c'est à l'inventeur plus qu'au savant que l'on doit les progrès techniques.

Un texte comme celui que nous reproduisons ici donne lieu à de multiples commentaires. Ne serait-ce que le titre, qui est l'occasion d'un exercice sur les aspects (Introduction en anglais : s'agit-il d'un progressif, d'un duratif, d'un inchoatif? Il faut choisir entre apparition, avènement, essor..., introduction en français ne pouvant être ici un cooccurrent de " recherche industrielle").

Tels sont quelques exemples de travaux pratiques préparés à domicile par les étudiants, discutés en classe par les étudiants et le professeur sur le mode rétroactif. L'autocorrection effectuée à l'issue des échanges oblige les étudiants à prendre des décisions concrètes en étant confrontés à leur texte. Enfin, le professeur contrôle l'autocorrection et rend les copies la semaine suivante. La boucle pédagogique rétroactive est ainsi bouclée.

\section{CONCLUSION}

Même si le décodage sémantique est souvent une pierre d'achoppement pour certains étudiants, l'essentiel du travail dans un cours de traduction générale anglais/ français porte sur la transparence du message en LA. Pour cette raison, ce cours est largement tributaire des quatre cours de soutien.

C'est un travail incessant d'affinage, de peaufinage de la langue ainsi que des connaissances culturelles, sans exhibitionnisme ni forfanterie. Comme on l'a souvent souligné, la bonne traduction est celle qui se fait oublier comme traduction et celle qui est conforme à l'état contemporain de la langue, sans trivialité ni purisme qui parasiteraient le message. L'enjeu ultime de ce cours est sans doute de convaincre l'apprentitraducteur qu'il doit acquérir une excellente "sensibilité linguistique " et que c'est sur cette sensibilité linguistique que le professeur et l'étudiant en traduction vont pouvoir travailler pour faire de ce dernier un traducteur compétent.

Notes

1. Jean-Paul Bénard et Paul Horguelin (1977) : Pratique de la traduction - Version générale, Montréal, Linguatech.

2. Jean Delisle (1980) : l'Analyse du discours comme méthode de traduction, Ottawa, Presses de l'Université d'Ottawa ; le Guide de la traduction appliquée (t. 1 : Version, Montréal, PUQ, 1978) de R. Larose, G. Vitale et $M$. Sparer mérite d'être cité parmi les bons ouvrages qui se situent dans notre optique.

3. Le concept de rétroaction (feedback) que nous empruntons à la pragmatique de la communication paraît propre à rendre compte de ce que nous tentons de réaliser en pédagogie de la traduction (cf. Paul Watzlawick et coll., Pragmatics of Human Communication, A Study of Interactional Patterns, Pathologies, and Paradoxes, New York, W.W. Norton, 1967). Le système interpersonnel qu'est le groupe étudiants/ professeur peut être vu comme une boucle rétroactive dans laquelle les informations injectées et régulées selon des paramètres de référence suivent des trajectoires diverses. Ces dernières sont continuellement et tour à tour soit centrifuges soit centripètes : le texte à traduire dans ses multiples dimensions et les possibilités linguistiques en LA sont les deux axes par lesquels passent lesdites trajectoires. Dans la mesurc où l'objectif est l'acquisition individuelle d'une technique (traduire) et, ultimement, la production d'un 
texte traduit, le rôle du professeur est "stabilisant " et en cela sa fonction dans la rétroaction est la recherche de l'équilibre de tous les éléments.

4. Ces critères ont été dégagés par Jean Darbelnet, puis repris par Paul Horguelin, notamment en révision professionnelle. Ils nous paraissent bien adaptés aux objectifs d'un premier cours de version générale.

5. Cette manière d'aborder un texte à traduire doit beaucoup à notre pratique de la traduction au dictaphone. Voir à ce propos notre article : "Esquisse d'une méthode de traduction au dictaphone ", META, 21:4 (décembre 1976). 